\title{
Papillon Lefèvre syndrome - Rare or Underdiagnosed?
}

\author{
Authors
}

\section{Dr Amit Chauhan ${ }^{1}$, Dr Ghanshyam Verma ${ }^{2}$, Dr Gita Ram Tegta ${ }^{3}$}

${ }^{1}$ Ex -Resident ${ }^{2}$ Associate Professor, ${ }^{3}$ Professor and Head of Department of Dermatology, venereology

Indira Gandhi Medical College Shimla HP. India

*Corresponding Author

Dr Ghanshyam Verma

Associate Professor Dept of Dermatology, Venereology Indira Gandhi Medical College Shimla HP. India

Email:drshyamverma77@gmail.com

\begin{abstract}
Papillon Lefèvre syndrome represents an autosomal recessive condition being characterised by palmarplantar hyperkeratosis and early onset of a severe destructive periodontitis leading to premature loss of both primary and permanent dentitions. Incidence varies from 1 to 4 persons per million. The available literature emphasises the need for a collaborative effort on the part of dermatologist as well as dentist for diagnosing this syndrome at an early age thus to prevent further complications. We report a case of Papillon Lefèvre syndrome along with treatment and follow up of the patient.
\end{abstract}

Keywords: Papillon-Lefèvre syndrome, Hyperkeratosis, Periodontitis.

\section{Introduction}

Papillon Lefèvre syndrome is an autosomal recessive disorder first described in 1924, by two French physicians Papillon and Lefevre. ${ }^{1}$ It is characterised by palmoplantar hyperkeratosis along with severe, early onset periodontitis and premature loss of primary and permanent teeth. The syndrome is believed to affect 1 to 4 persons per million. ${ }^{2}$ Genetics involve mutation of gene on long arm of chromosome 11 (11q14) regulating production of enzyme cathespin C. ${ }^{3,4}$ Cutaneous changes include well-demarcated erythematous hyperkeratotic lesions on the palms, dorsum of the hands, soles and also the interphalangeal joints. These plaques occur focally, but usually involve the entire surface of the palms and soles, sometimes extending onto the dorsal surfaces of the hands and feet posing a diagnostic dilemma for the dermatologists to differentiate it from other hereditary and acquired palmoplantar keratodermas. The key to differentiate this syndrome from other types of palmoplantar keratoderma lies in the associated periodontopathy and premature loss of primary and permanent dentition. Peridontal disease usually has its onset between the ages of 1 to 4 years. The primary (deciduous) teeth frequently become loose and fall out. Without treatment, most of the secondary (permanent) teeth may also be lost. ${ }^{3,4}$ But the oral findings are sometimes missed by the dermatologists thus posing a diagnostic dilemma. Associated features may include intra-cranial calcifications, susceptibility to bacterial infections and mental retardation. ${ }^{5}$

\section{Case Report}

We report a case of 17 years old female who presented with the chief complaint of fissuring and thickening of hands and feet for five years of age 


\section{JMSCR Vol||07||Issue||04||Page 836-839||April}

along with itching. On examination there were wellcircumscribed, erythematous, scaly, indurated non tender plaques affecting the skin of her palms and soles bilaterally and extending onto the dorsal surfaces (Figure a, b) i.e transgradience was there. Her nails and hair were normal. There was no history suggestive of hyperhidrosis.

There was history of loss of primary teeth by 4 years of age and loss of permanent teeth by 15 years of age with just 5 teeth remaining. Intraoral examination showed that the patient had lost most of her permanent teeth and the remaining teeth that is four premolars and one molar showed grade II mobility with heavy deposits of plaque and calculus. (Figure c) Orthopantogram showed severe peridontal destruction and partial edentulism along with severe bone loss both in maxilla and mandible with floating in air appearance, thus was consistent with findings of Papillon-Lefèvre Syndrome (Figure d).

Complete blood count, serum calcium and alkaline phosphatase levels were done and were found to be within normal limits. On the basis of history, clinical and radiographical features a diagnosis of Papillon-Lefevre syndrome was made.

Emollients with oral Isotretinoin was started. After 8 weeks, there was marked improvement with reduction of keratoderma (Figure e). Oral hygiene by mouth rinses twice daily and extraction along with replacement of remaining teeth with denture was planned by department of prosthodontics.

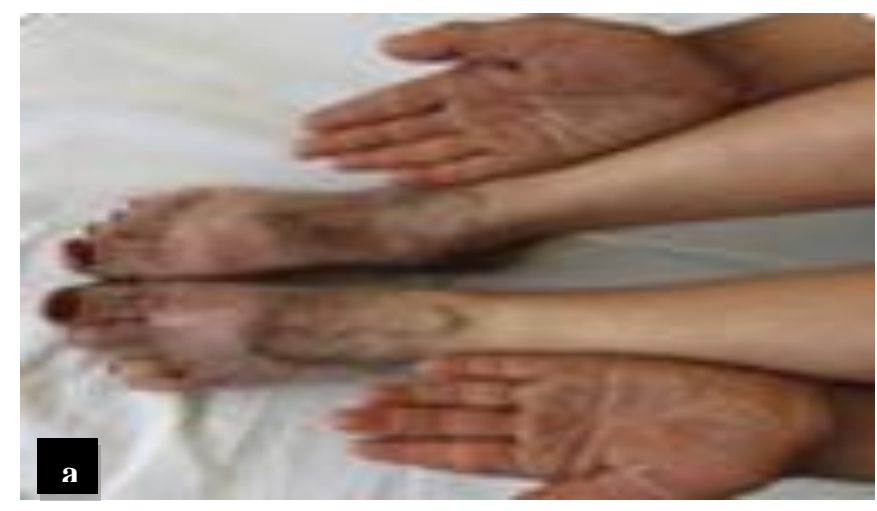

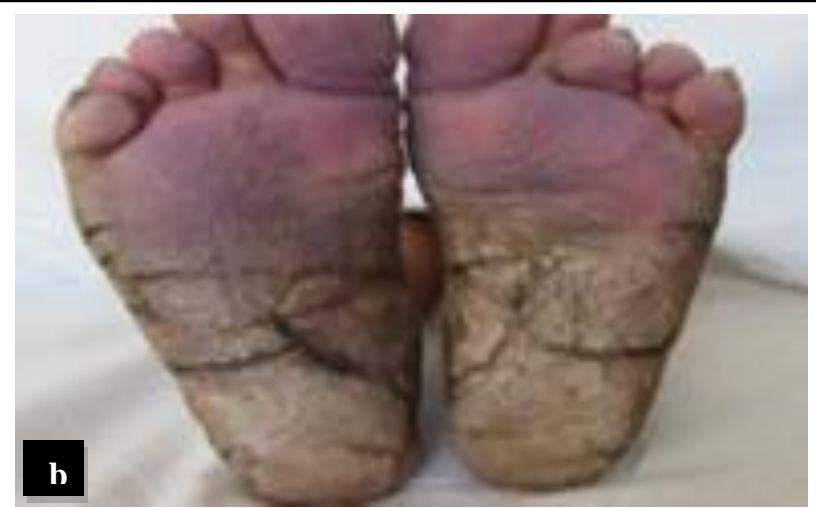
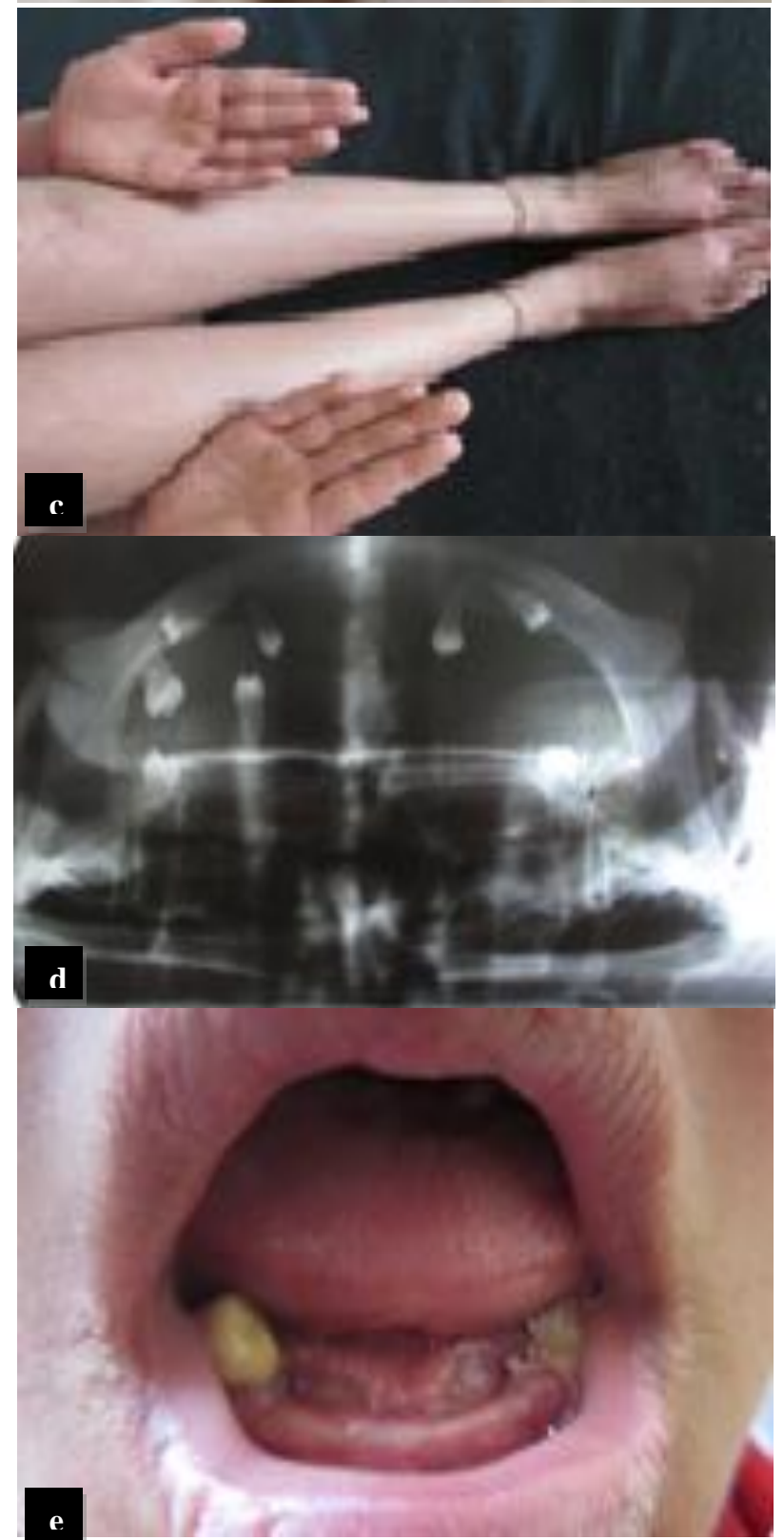

Figure (a)(b) Symmetrical, well-demarcated, erythematous, hyperkeratotic, scaly plaques on palm and soles with transgradience,(c) Marked improvement with retinoids after 8 weeks. (d) Orthopantogram - Severe peridontal destruction around all premolars and molars with floating in air appearance. 18 and 28 in eruption phase, (e) Premature loss of permanent teeth. 


\section{Discussion}

PLS is a disorder of keratinization, ${ }^{6}$ which manifests as palmoplantar hyperkeratosis along with severe destructive periodontitis affecting both the deciduous as well as the permanent dentitions. Other manifestations like calcification of the falx cerebri and dura, pyogenic infections, nail dystrophy and generalized hyperhidrosis may also be associated. ${ }^{6}$ The most severely affected regions in PLS are the keratinized gingivae of oral cavity and skin of palms and soles. The pathogenesis of PLS involve mutations of the cathepsin-C gene located on the 11q14-q21 region of the chromosome, encoding a lysosomal protease in the interval between D11S4082 and D11S931. ${ }^{7}$ The cathepsin C gene is expressed in the keratinized oral gingiva and epithelial region (palms, soles, knees) which is important in the structural growth and development of skin. Mutations in this gene lead to almost total loss of cathepsin $\mathrm{C}$ activity in patients with PLS. ${ }^{8,9,10}$ Thus impaired function of cathepsin C gene in epithelial regions lead to abnormalities like thickening of the palms and soles. Thus mutation in lysosomal protease enzyme which otherwise plays a pivotal role in maintaining balance between oral microflora and immune system by protein degradation and activation of proenzyme, leads to altered host response and hence failure of bacterial clearance from the gingiva causing destructive periodontosis resulting in tooth loss.

The differential diagnosis related to this syndrome includes acrodynia, hypophosphatasia, and cyclic neutropenia. Presence of erythrocyanosis, insomnia, muscle pain, tachycardia, psychic disturbances, and premature eruption of teeth with dystrophic enamel differentiates it from acrodynia and mercury poisoning. Hypophosphatasia is differentiated from PLS by the clinical features of enlarged wrists, knock-knee, bowing of the femur and the tibia, hypoplastic teeth, along with increased amounts of phosphoethanolamine in the urine. In cyclic neutropenia, the hyperkeratosis of palms and soles is absent. The other differentials are Howel-Evans syndrome, Greither syndrome, and keratosis punctata but periodontopathy is not seen in them. ${ }^{11}$
A multidisciplinary approach involving a team of dermatologist, pediatrician, and dental surgeon is important in overall care of the patient with PLS. Dermatological manifestations of PLS are mostly treated with emollients, salicylic acid and topical steroids. Oral retinoids such as acitretin, isotretinoin and etretinate are proven to be beneficial in treating both dental and cutaneous lesions of PLS. ${ }^{12}$ These retinoids are vitamin A metabolites which regulate the growth and differentiation of epithelial cells, and thus exert a profound effect on keratinization by decreasing the total keratin content of keratinocytes. More recently, systemic therapy with synthetic retinoids has been found to be effective in treating cutaneous lesions in patients with PLS. ${ }^{13,14}$ Retinoids are also thought to influence inflammation by stimulating both humoral and cellular immunity. In our case also, after 8 weeks of oral isotretinoin, there was significant improvement with marked reduction of keratodermas. The aim of dental treatment in PLS is to eliminate the reservoir of causative organisms and thus to control destruction of periodontium. Retinoid treatment should be started at the time of eruption of successor teeth and continued till the completion of the normal development process. ${ }^{15}$ Several treatment modalities have been shown to be effective such as: scaling and root planning; oral hygiene instructions and mouth rinses with $0.2 \%$ chlorhexidine gluconate; and a course of antibiotic treatment therapy, which is suggested to control the active periodontitis. Extraction of deciduous teeth with poor prognosis and eradicating the periodontal pathogens creates a safe environment for eruption of permanent teeth. To restore masticatory function, prosthetic approach is necessary. Thus, a collaborative effort on the part of dermatologist and dentist helps in decreasing patient's morbidity. Missing of the oral findings will miss the diagnosis, thus there is a need to bridge the gap among different fields for correct diagnosis and management of patients. 


\section{References}

1. Muppa R, Prameela B, Duddu M, Dandempally A. Papillon-lefevre syndrome: A combined approach from the dermatologist and dentist - A clinical presentation. Indian J Dermatol 2011; 56:740-3.

2. Hart TC, Shapira L. Papillon-Lefèvre syndrome. Periodontol 2000 1994; 6:88-100.

3. Dhanrajani PJ. Papillon-Lefevre syndrome: Clinical presentation and a brief review. Oral Surg Oral Med Oral Pathol Oral RadiolEndod 2009;108: e1-7.

4. Yang Y, Bai X, Liu H, Li L, Cao C, Ge L. Novel mutations of cathepsin $\mathrm{C}$ gene in two Chinese patients with Papillon-Lefèvre syndrome. J Dent Res 2007; 86:735-8.

5. Kansky AA, Potocnik M, Kansky A. Papillon-Lefèvre syndrome. Acta Dermatovenerological A 1997; 6:60-5.

6. KordValeshabad A, Mazidi A. Papillonlefèvre syndrome: A series of six cases in the same family. ISRN Dermatol 2012; 2012:139104.

7. Thakker N. Genetic analysis of Papillon Lefevre syndrome. Oral Dis. 2000; 6:263.

8. Angel TA, Hsu S, Kornbleuth SI, Kornbleuth J, Kramer EM. Papillon-Lefevre syndrome: A case report of four affected siblings. J Am Acad Dermatol 2002;46: S810.

9. Allende LM, Moreno A, de Unamuno P. A genetic study of cathepsin $\mathrm{C}$ gene in two families with Papillon-Lefèvre syndrome. Mol Genet Metab. 2003; 79:146-8.

10. Umeda M, Zhang VJ, Koseki T, Ishikawa I. Clinical, bacteriological and immunological examination and treatment of two PapillonLefèvre syndrome patients. Kokubyo Gakkai Zasshi. 1990;57(3):430-440.

11. Nagaveni NB, Suma R, Shashikiran ND, Subba Reddy VV. The Papillon-Lefevre syndrome: Report of two cases in the same family. J Indian Soc PedodPrev Dent. 2008; $26: 78-81$.
12. Ullbro C, Brown A, Twetman S. Preventive periodontal regimen in Papillon-Lefèvre syndrome. Pediatr Dent 2005; 27:226-32.

13. Singh R, Nor M, Ghazali W. Atypical Papiilon-Lefèvre syndrome: keratosis palmoplantaris with periodontopathy. Int $\mathbf{J}$ Dermatol. 1993; 32:450-52.

14. BerUllbro C, Crossner CG, Nederfors T, Alfadley A, Thestrup-Pedersen K. Dermatologic and oral findings in a cohort of 47 patients with Papillon-Lefèvre syndrome. J Am Acad Dermatol 2003; 48:345-51.

15. Singla A, Sheikh S, Jindal SK, Brar R. Papillon Lefevre syndrome: Bridge between dermatologist and dentist. J Clin Exp Dent 2010;2:e43-6. 